\title{
The Effectiveness of 6 Months Hydrotherapy Program Based on Halliwick Concept on the Respiratory System of Down Syndrome Children
}

\author{
Konstantinos Chandolias ${ }^{*}$ (D), Eliza Konstantinidou², Efthymios Bikis ${ }^{3}$, Anna Hourlia4, \\ Thomas Besios 5 , George Tsigaras 6 \\ ${ }^{1}$ Department of Physical Therapy, University of Thessaly, Lamia, Greece \\ ${ }^{2}$ Rehabilitation Center Animus, Larissa, Greece \\ ${ }^{3}$ Physiotherapy Practice, Larissa, Greece \\ ${ }^{4}$ Speech and Language Therapy Practice, Larissa, Greece \\ ${ }^{5}$ Department of Physical Therapy, University of Thessaly, Lamia, Greece \\ ${ }^{6}$ Hippokratio General Hospital, Thessaloniki, Greece \\ Email: *konchand@uth.gr
}

How to cite this paper: Chandolias, K., Konstantinidou, E., Bikis, E., Hourlia, A., Besios, T. and Tsigaras, G. (2021) The Effectiveness of 6 Months Hydrotherapy Program Based on Halliwick Concept on the Respiratory System of Down Syndrome Children. Journal of Biosciences and Medicines, 9, 20-26.

https://doi.org/10.4236/jbm.2021.93003

Received: February 14, 2021

Accepted: March 15, 2021

Published: March 18, 2021

Copyright $\odot 2021$ by author(s) and Scientific Research Publishing Inc. This work is licensed under the Creative Commons Attribution International License (CC BY 4.0).

http://creativecommons.org/licenses/by/4.0/ (c) (i) Open Access

\begin{abstract}
The aim of the study was to investigate the effect of a hydrotherapy program on FVC, FEV, PEF, RR and $\mathrm{SaO}_{2}$ on children with Down syndrome over six months and to compare it with a conventional respiratory physiotherapy program. Eighteen children, with Down Syndrome, aged 6 - 11 years $(9.53 \pm$ 0.454 ), divided into two groups of nine, the intervention group (IG), that participated in the hydrotherapy program and the control group (CG) participated in the classical physiotherapy program. We calculated mean values of FVC, FEV, PEF, RR and $\mathrm{SaO}_{2}$ before and after six months intervention for both groups. There was a statistically significant improvement in all factors for both groups. However, were statistically more significant for the intervention group (IG). Based on a specific protocol of intervention in the water and at the same time with a group of children who participated in a similar program of classical respiratory physiotherapy, it was found to be statistically more important than the second group in improving respiratory function. We recommend the use of hydrotherapy as a complementary therapy that should be part of the weekly program of these children in addition to the existing treatments they attend.
\end{abstract}

\section{Keywords}

Hydrotherapy, Physical Therapy, Respiratory, Halliwick, Down Syndrome 


\section{Introduction}

The increasing of life expectancy of people with Down syndrome (DS), results in phenomena of comorbidity, and the natural history of the health status bear over the years. For this reason, attempts have been made to promote a better quality of life and greater social inclusion of this population through rehabilitation strategies tailored and targeted to the specific needs of people with DS [1]. DS has an incidence of 1:660 live births [2]. It is considered a common chromosomal abnormality, caused by the presence of an extra chromosome. The presence of the syndrome results in variable degrees of delayed motor development, physical and mental. Infections of the upper airways and/or pneumonia cases come to affect $77.0 \%$ of patients with DS [3].

The international literature it appears that people with Down syndrome show reduced frequency and intensity of physical activity compared to the average [4].

In addition, although routine rehabilitation programs focus on training the respiratory muscles, weaknesses of these muscles have been reported in people with DS when compared to typical development individuals of the same age and gender [5]. This is an extremely important finding, since respiratory muscle weakness is associated with the presence of airway diseases, which are one of the leading causes of recurrent illnesses in this population [1].

Children with Down syndrome have been reported with upper and lower airway abnormalities including a small upper airway, decreased numbers of alveoli and reduced surface area [6]. These major problems can lead to decreased lung volume and this may be a key factor that leads to poor level of physical activity.

The philosophy of Halliwick concept was developed by James McMillan in England and aims to teach water independence to people with disabilities, while considering the specific characteristics of the aquatic environment [7]. Halliwick Concept follows the Ten Point Program. The first 2 points, mental adaptation, and release are elements that are constantly trained as the parallel and physical skills develop. Mental adaptation refers to the development of comfort in the aquatic environment. One aspect that emphasizes Halliwick concept is the ability to control breathing to relax mentally and physically. This focus on the psychological state of the individual creates a real and perceived safe learning environment where the swimmer can gain confidence in his own abilities in the water. Release is the process of gaining physical and mental independence, so that the swimmer gains control of their movement in the water. There are studies that have proven the positive effect of hydrotherapy Based on Halliwick Concept in children with cerebral palsy [8].

\section{Methods}

\subsection{Design}

This study was a randomized controlled trial. All children and parents who participated in this study, after being thoroughly informed about all the study procedures, signed a consent form. The research protocol was implemented in ac- 
cordance with the Declaration of Helsinki (1975), as revised in 2013.

Approval was required from the Internal Ethics Committee of the Department of Physical Education and Sports Science, University of Thessaly. The research sample was confiscated and approved with protocol number 3-3/15/07/2015.

\subsection{Subjects}

Eighteen children from both genders, with Down Syndrome, aged 6 - 11 years $(9.53 \pm 0.454)$, diagnosed by karyotype. We excluded individuals with severe mental disabilities and understanding of performing techniques and with a diagnosis of not-treated congenital heart defects. Anthropometric characteristics of the subjects are shown in Table 1.

\subsection{Equipment}

The respiratory function was measured with a spirometer, a cardiovascular blood flow-meter and a pulse oximeter. The volume of air exhaled at the end of the first second of forced expiration (FEV), forced vital capacity (FVC), forced expiratory volume in the first second $(\mathrm{FEV})$, respiratory rate $(\mathrm{RR})$ a CareFusion Pulmolife spirometer. Subsequently, the saturation of oxygen $\left(\mathrm{SPO}_{2}\right)$ and the heart rate (HR) were measured with a GIMAOXY-4 oximeter. Finally, with the CareFusion Micro Peak flow meter, the peak expiratory flow rate (PEF) was measured.

\subsection{Procedures}

The subjects were divided into two groups of nine individuals. The intervention group (IG) which was the group that participated in the hydrotherapy program and the control group (CG) which participated in the classical physiotherapy program. All subjects in both groups were evaluated using instruments for the values of FVC, FEV, PEF, RR and $\mathrm{SaO}_{2}$ before and after six months intervention.

\subsection{Interventions}

Both groups of children who participated in the study participated in an intervention program lasting 6 months and frequency twice a week. In both intervention programs, either in water or on land, the main purpose of the intervention was respiratory function, and the organization of the treatment session was breathing, respiratory muscles and physical condition.

Intervention Group: The hydrotherapy sessions were based on the Halliwick

Table 1. Anthropometric characteristics of the subjects.

\begin{tabular}{cc}
\hline Characteristics & Subjects $(\mathbf{N}=18)$ \\
\hline Age (year) & $9.53 \pm 0.454$ \\
Weight $(\mathrm{kg})$ & $38.6 \pm 6.31$ \\
Height $(\mathrm{cm})$ & $1.31 \pm 0.23$ \\
BMI $\left(\mathrm{kg} / \mathrm{m}^{2}\right)$ & $23.01 \pm 2.33$ \\
\hline
\end{tabular}


Concept and composed of activities in standing, back float and prone float position with water at $110 \mathrm{~cm}$ depth and water temperature between $33^{\circ} \mathrm{C}$ and $34^{\circ} \mathrm{C}$. The intervention program focused on exhalation, blowing, normalizing breathing pattern, underwater exhalation in combination with chest deployment activities, upper extremity and torso strengthening and endurance activities.

Control group: The control group followed a program of classical respiratory physiotherapy. The program focused on breathing exercises from various positions, exhalation, breathing, rhythm and at the same time exercising the respiratory muscles and improving physical endurance and fitness.

\subsection{Statistical Analysis}

The statistical package SPSS 21 was used for data analysis. We calculated mean values of FVC, FEV, PEF, RR and $\mathrm{SaO}_{2}$ before and after six months intervention for both groups. The results were compared using the paired-t-test to determine the level of significance. The comparison between the two groups was performed using the independent $\mathrm{t}$-test $(\mathrm{p}<0.05)$.

\section{Results}

The research results are presented in Tables 2-4. From the tables, it appears that there is a statistically significant improvement in FVC, FKV, PEF, RR, $\mathrm{SaO}_{2}$ for both groups. However, comparing the results of the intervention group with the control group, the improvement of the factors is statistically more significant for the intervention group during the six months.

Table 2. The difference between the pre- and post-test values of the FVC, FEV, PEF, RR and $\mathrm{SaO}_{2}$ of the intervention group.

\begin{tabular}{ccccc}
\hline & \multicolumn{2}{c}{ Mean + SD } & & t-value \\
\cline { 2 - 3 } & \multicolumn{1}{c}{ Pre } & Post & Significant \\
\cline { 2 - 3 } FVC & $1.47 \pm 0.41$ & $2.99 \pm 0.46$ & 5.46 & $<0.05$ \\
$\mathrm{FEV}$ & $1.12 \pm 0.26$ & $2.52 \pm 0.33$ & 4.634 & $<0.05$ \\
$\mathrm{PEF}$ & $2.05 \pm 0.72$ & $3.25 \pm 0.78$ & 5.512 & $<0.05$ \\
$\mathrm{RR}$ & $24.51 \pm 2.82$ & $20.01 \pm 2.02$ & 4.83 & $<0.05$ \\
$\mathrm{SaO}_{2}$ & $89.52 \pm 3.46$ & $97.21 \pm 3.34$ & 5.17 & $<0.05$ \\
\hline
\end{tabular}

Table 3. The difference between the pre- and post-test values of the FVC, FEV, PEF, RR and $\mathrm{SaO}_{2}$ of the control group.

\begin{tabular}{|c|c|c|c|c|}
\hline & \multicolumn{2}{|c|}{ Mean + SD } & \multirow{2}{*}{ t-value } & \multirow{2}{*}{ Significant } \\
\hline & Pre & Post & & \\
\hline FVC & $1.34 \pm 0.42$ & $2.34 \pm 0.36$ & 2.22 & $<0.05$ \\
\hline FKV & $1.22 \pm 0.36$ & $1.88 \pm 0.32$ & 2.36 & $<0.05$ \\
\hline PEF & $2.18 \pm 0.54$ & $2.61 \pm 0.51$ & 2.43 & $<0.05$ \\
\hline $\mathrm{RR}$ & $25.81 \pm 2.53$ & $23.01 \pm 2.53$ & 2.54 & $<0.05$ \\
\hline $\mathrm{SaO}_{2}$ & $90.07 \pm 3.24$ & $93.41 \pm 3.32$ & 3.45 & $<0.05$ \\
\hline
\end{tabular}


Table 4. The difference between the values of the FVC, FEV, $\mathrm{RR}$ and $\mathrm{SaO}_{2}$ in the intervention and the control group.

\begin{tabular}{ccccc}
\hline & \multicolumn{2}{c}{ Mean + SD } & t-value & Significant \\
\cline { 2 - 3 } & $\begin{array}{c}\text { Intervention } \\
\text { Group }\end{array}$ & $\begin{array}{c}\text { Control } \\
\text { Group }\end{array}$ & & \\
\hline $\mathrm{FVC}$ & $2.99 \pm 0.46$ & $2.34 \pm 0.36$ & 2.59 & $<0.05$ \\
$\mathrm{FKV}$ & $2.52 \pm 0.33$ & $1.88 \pm 0.32$ & 2.48 & $<0.05$ \\
$\mathrm{PEF}$ & $3.25 \pm 0.78$ & $2.61 \pm 0.51$ & 2.47 & $<0.05$ \\
$\mathrm{RR}$ & $20.01 \pm 2.02$ & $23.01 \pm 2.53$ & 2.34 & $<0.05$ \\
$\mathrm{SaO}_{2}$ & $97.21 \pm 3.34$ & $93.41 \pm 3.32$ & 2.87 & $<0.05$ \\
\hline
\end{tabular}

\section{Discussion}

This study investigated the change in respiratory function in children with Down syndrome. The authors assessed the respiratory muscle strength of 54 individuals with DS before and after a rehabilitation program consisting of 10 hydrotherapy sessions. The authors used the Manovacuometry method to indirectly assess the strength of the respiratory muscle (maximal respiratory pressure and maximal expiratory pressure). They observed that the program resulted in an increase in maximal expiratory pressure but could not significantly increase maximal respiratory pressure. Thus, the authors conclude that hydrotherapy using the Bad Ragaz method is a beneficial method for treating people with DS. In the reported study [9] interesting results are presented, which can be partly taken as a basis for clinical practice, as hydrotherapy can be a fun way to treat patients with DS, providing resistance due to the disorder and thus allowing muscle strengthening.

It is stated that hydrotherapy is a method that can offer both physical and psychosocial and mental improvements in people with DS [10]. These improvements are due, among other things, to the positive effect that hydrotherapy has on the respiratory system of people with Down syndrome. However, care must be taken when interpreting the study data, as the test used to assess muscle strength is not considered an absolute standard for this measurement, and therefore may not have recorded differences between assessments.

In addition, Manovacuometry is an indirect measure of respiratory muscle strength, which may have affected the results. To demonstrate the effects of Bad Ragaz hydrotherapy on respiratory muscle strength in people with DS, it would be extremely interesting to conduct a study with a control group, using more specific measurements (such as the dynamometer, for example) to it is possible to use scientific data in clinical practice.

Another study aimed to investigate the effects of hydrotherapy on respiratory function in patients with Down syndrome. The sample consisted of 7 people with Down syndrome, aged between 12 and 37 years (24 \pm 8 years) where $71.4 \%$ were women. Participants underwent a comprehensive hydrotherapy treatment. Evaluations included the use of a manometer, maximum expiratory flow, and a 
one-minute sit-up test. The hydrotherapy treatment lasted one hour, once a week for ten weeks. The analysis of the results showed that the treatment had a positive effect on the increase of the maximum inspiratory pressure $(51 \pm 13$ to $\left.59 \pm 11 \mathrm{~cm} \mathrm{H} \mathrm{H}_{2} \mathrm{O}, \mathrm{P}=0.06,95 \% \mathrm{CI}\right)$ and an improvement in the values of the expiratory maximal pressure $(207 \pm 54$ to $243 \pm 71, \mathrm{P}=0.01,95 \% \mathrm{CI})$ [11].

In the present study, an attempt was made to investigate how a specific hydrotherapy program based on specific steps through the Halliwick Concept can influence the development and improvement of the respiratory function of children with Down syndrome. Based on a specific protocol of intervention in the water and at the same time with a corresponding group of children who participated in a similar program of classical respiratory physiotherapy, it was found to be statistically more important than the second group in improving respiratory function. This was in line with existing research and enhances the effect of hydrotherapy as an additional contribution to strengthening the respiratory system of these children.

\section{Conclusion}

Based on the results of this study, we recommend that the inclusion of children with Down Syndrome in programs aimed at improving respiratory function and endurance is particularly important. Given the statistically significant outcome of the water group, we recommend the use of hydrotherapy as a complementary therapy that should be part of the weekly program of these children in addition to the existing treatments they attend.

\section{Acknowledgements}

We gratefully acknowledge the parents of the children and the physiotherapists for their participation.

\section{Conflicts of Interest}

The authors report no conflicts of interest.

\section{References}

[1] Tenenbaum, A., Chavkin, M., Wexler, I.D., Korem, M. and Merrick, J. (2012) Morbidity and Hospitalizations of Adults with Down Syndrome. Research in Developmental Disabilities, 33, 435-441. https://doi.org/10.1016/j.ridd.2011.09.026

[2] Devlin, L. and Morrison, P.J. (2004) Accuracy of the Clinical Diagnosis of Down Syndrome. Ulster Medical Journal, 73, 4-12.

[3] Bertelli, E.C.P., Biselli, J.M. and Bonfim, D. (2009) Clinical Profile of Children with Down Syndrome Treated in a Genetics Outpatient Service in the Southeast of Brazil. Revista da Associação Médica Brasileira, 55, 547-552. https://doi.org/10.1590/S0104-42302009000500017

[4] Gupta, S., Rao, B.K. and Kumaran, S.D. (2011) Effect of Strength and Balance Training in Children with Down's Syndrome: A Randomized Controlled Trial. Clinical Rehabilitation, 25, 425-432. https://doi.org/10.1177/0269215510382929 
[5] Da Silva, V.Z.M., de França Barros, J., de Azevedo, M., de Godoy, J.R.P., Arena, R. and Cipriano Jr., G. (2010) Bone Mineral Density and Respiratory Muscle Strength in Male Individuals with Mental Retardation (with and without Down Syndrome). Research in Developmental Disabilities, 31, 1585-1589. https://doi.org/10.1016/j.ridd.2010.05.003

[6] McDowell, K. and Craven, D. (2011) Pulmonary Complications of Down Syndrome during Childhood. The Journal of Pediatrics, 158, 319-325.

https://doi.org/10.1016/j.jpeds.2010.07.023

[7] McMillan, J. (1978) The Role of Water in Rehabilitation. Fysioterapeuten, 45, 87-90.

[8] Christodoulaki, E., Chandolias, K. and Hristara-Papadopoulou, A. (2018) The Effect of Hydrotherapy: Halliwick Concept on the Respiratory System of Children with Cerebral Palsy. BAOJ Pediat, 4, 63.

[9] Castoldi, A., Périco, E. and Grave, M. (2012) Avaliação da força muscular e capacidade respiratória em pacientes com síndrome de Down após Bad Ragaz. Revista Neurociências, 20, 386-391. https://doi.org/10.4181/RNC.2012.20.740.6p

[10] Campion, M.R. (1997) Hydrotherapy: Principles and Practice. Butterworth-Heinemann.

[11] Braga, H.V., Dutra, L.P., Veiga, J.M. and Junior, E.P.P. (2019) Efeito da fisioterapia aquática na força muscular respiratória de crianças e adolescentes com Síndrome de Down. Arquivos de Ciências da Saúde da UNIPAR, 23, 9-13. https://doi.org/10.25110/arqsaude.v23i1.2019.6392 